\title{
EXTENSIONS OF LEFT UNIFORMLY CONTINUOUS FUNCTIONS ON A TOPOLOGICAL SEMIGROUP
}

\author{
SAMUEL J. WILEY 1
}

\begin{abstract}
For any topological semigroup $S$ with separately continuous operation, let $C(S)$ denote the set of all bounded continuous real valued functions on $S$ with the supremum norm and let $\operatorname{LUC}(S)$ denote the set of all $f$ in $C(S)$ such that whenever $\{s(\gamma)\}$ is a net in $S$ which converges to some $s$ in $S$, then $\sup \{|f(s(\gamma) t)-f(s t)|: t \in S\}$ converges to 0 . In this paper we prove that if $S$ is an abelian subsemigroup of a compact topological group and $f \in \operatorname{LUC}(S)$, then there is an $F \in \operatorname{LUC}(G)$ where $F(s)=f(s)$ for all $s \in S$. We also show whenever there is an extension of the type indicated above, there is a norm preserving extension.
\end{abstract}

1. Introduction. By a topological semigroup we mean a semigroup with a Hausdorff topology in which the product $s t$ is separately continuous. If $X$ is a topological space, $C(X)$ indicates the algebra of all bounded continuous real valued functions on $X$ with the supremum norm. If $S$ is a topological semigroup, $l_{s}: C(S) \rightarrow C(S)$ is defined by $l_{s} f(t)=f(s t)$ and $r_{s}: C(S) \rightarrow C(S)$ is defined by $r_{s} f(t)=f(t s)$. Let $S$ be a topological semigroup. $f \in C(S)$ is said to be left uniformly continuous or $f \in \operatorname{LUC}(S)$ when the function $\theta: S \rightarrow C(S)$ defined by $\theta(s)=l_{s} f$ is continuous on $S$. The space $\operatorname{LUC}(S)$ is a closed subalgebra of $C(S)$ which contains the constants [8]. Mitchell [7] considered topological semigroups $T$ and subsemigroups $S$. He showed if $S$ is dense in $T$ and $\operatorname{LUC}(S)$ has a left invariant mean, so has $\operatorname{LUC}(T)$. In the next section we will show that if $S$ is an abelian subsemigroup of a compact topological group, then every $f \in \operatorname{LUC}(S)$ has an extension to an $F \in \mathrm{LUC}(T)$.

If $(X, \mathbb{U})$ is a uniform space, then $\mathrm{UC}(X)$ denotes the set of all bounded real valued functions on $X$ which are uniformly continuous with respect to $\mathscr{U}$. In the following proposition the equivalence of (a) and (b) follows from

Presented in part to the Society, April 9, 1971; received by the editors August 16, 1971. AMS 1970 subject classifications. Primary 22A99, 22C05; Secondary 46J10.

Key words and phrases. Extensions, topological semigroups, compact groups, norm preserving extensions, uniformly continuous functions.

${ }^{1}$ Part of this paper was included in a doctoral dissertation written at Temple University, 1970, under the direction of Dr. Theodore Mitchell. The author is most indebted to Dr. Mitchell for his valuable suggestions and encouragements during the preparation of the dissertation.

c. American Mathematical Society 1972 
Kelley [5, p. 86], (b) implies (c) is found in Granirer and Lau [2, Lemma $3]$, the rest comes directly from the definitions.

Proposition 1. Let $S$ be a topological semigroup and $f \in C(S)$. The following are equivalent.

(a) $f \in \operatorname{LUC}(S)$.

(b) For every $s \in S$, if $\{s(\gamma)\}$ is a net converging to $s$, then $l_{s(\gamma)} f$ converges to $l_{s} f$ uniformly.

(c) $\left\{r_{t} f: t \in S\right\}$ is an equicontinuous family of functions on $S$.

If $S$ is a topological group, then (a), (b) and (c) are equivalent to (d).

(d) $f \in \mathrm{UC}(S)$ where the uniformity on $S$ is the usual right uniformity on a topological group [1, p. 243].

2. Extension theorems. It will be convenient to have the following definition. Let $S, T$ be topological semigroups, $S$ a subsemigroup of $T$. Then we will say the pair $(S, T)$ has property $P$, if for every $f \in \operatorname{LUC}(S)$ there is an $F \in \operatorname{LUC}(T)$ such that $\left.F\right|_{S}=f .\left.F\right|_{S}$ indicates the restriction of $F$ to $S$.

Proposition 2. Let $G$ be a topological group with the right uniformity and $S$ a subsemigroup of $G$ with the restriction uniformity. The following are equivalent.

(a) The pair $(S, G)$ has property $P$.

(b) $\operatorname{LUC}(S) \subset \mathrm{UC}(S)$.

(c) $\operatorname{LUC}(S)=\mathrm{UC}(S)$.

Proof. (a) $\rightarrow$ (b) If $f \in \operatorname{LUC}(S)$, then $f=\left.F\right|_{S}$ where $F \in \operatorname{LUC}(G)=\operatorname{UC}(G)$. Hence $\left.f \in \mathrm{UC}(G)\right|_{S} \subset \mathrm{UC}(S)$.

(b) $\rightarrow$ (c) If $f \in \mathrm{UC}(S)$, by Katetov [4, Theorem 3], $\left.f \in \mathrm{UC}(G)\right|_{S}=\left.\mathrm{LUC}(G)\right|_{S}$. Hence, by Lau [6, Proposition 1.3.1], $f \in \operatorname{LUC}(S)$.

(c) $\rightarrow$ (a) If $f \in \operatorname{LUC}(S)=\mathrm{UC}(S)$, by Katetov [4, Theorem 3] there is an $F \in \mathrm{UC}(G)=\operatorname{LUC}(G)$ such that $\left.F\right|_{S}=f$.

The following theorem is an immediate consequence of Propositions 1 and 2.

THEOREM 3. If $G$ is a topological group and $G^{\prime}$ is a subgroup of $G$, then the pair $\left(G^{\prime}, G\right)$ has property $P$.

The following example shows that we do not always have the extension property from a subsemigroup of a topological group to the group.

EXAMPLE. Let $G$ be the real numbers under addition, $S$ be the positive real numbers and $f(x)=\sin (1 / x)$. $f$ is not uniformly continuous on $S$ since it does not have a continuous extension to $\operatorname{cl}(S)$. But $f \in \operatorname{LUC}(S)$. So the pair $(S, G)$ does not have property $P$. We can see $f \in \operatorname{LUC}(S)$ since if 
$\{s(n)\}$ is a net in $S$ converging to $s \in S$, then

$$
\begin{aligned}
\left|l_{s(n)} f(x)-l_{s} f(x)\right| & =\left|\sin \frac{1}{s(n)+x}-\sin \frac{1}{s+x}\right| \\
& =\left|2 \sin \frac{1}{2}\left(\frac{1}{s(n)+x}-\frac{1}{s+x}\right) \cos \frac{1}{2}\left(\frac{1}{s(n)+x}+\frac{1}{s+x}\right)\right| \\
& \leqq 2\left|\sin \frac{1}{2} \frac{s-s(n)}{(s(n)+x)(s+x)}\right| .
\end{aligned}
$$

But $s$ is positive, so for sufficiently large $n, s(n)>\delta$ and $s>\delta$ where $\delta>0$. Since $x>0, s(n)+x>\delta$ and $s+x>\delta$. So

$$
\left|\frac{s-s(n)}{(s(n)+x)(s+x)}\right|<\left|\frac{s-s(n)}{\delta^{2}}\right| \text { for all } x \in S .
$$

Since the sine function is continuous and $(s-s(n)) / \delta^{2}$ converges to 0 , there is an $n_{0}$ such that for all $n \geqq n_{0}$,

\section{So $f \in \operatorname{LUC}(S)$.}

$$
2\left|\sin \frac{1}{2} \frac{s-s(n)}{(s(n)+x)(s+x)}\right|<\varepsilon \text { for all } x \in S .
$$

Mitchell [7, pp. 640-641] has given an example of a compact topological semigroup $T$ and a dense subsemigroup $S$ of $T$ where the pair $(S, T)$ does not have property $P$. We will now show that we have the desired extension whenever $T$ is a compact topological group and $S$ is abelian.

THEOREM 4. If $G$ is a compact topological group and $S$ is an abelian subsemigroup of $G$, then the pair $(S, G)$ has property $P$.

Proof. (i) Since $\operatorname{cl}(S)$ is a compact cancellation semigroup, it is a topological group [3, Theorem 9.16]. Hence by Theorem 3 we may assume $\operatorname{cl}(S)=G$.

(ii) Let $f \in \operatorname{LUC}(S), x(\alpha)$ be a net in $S$ which converges to $e$ and $a \in S$. Then $x(\alpha) a$ converges to $a$. Hence $\lim _{\alpha} f(x(\alpha) a y)=f(a y)$ uniformly for all $y$ in $S$.

(iii) Now since $G$ is compact, there is a unique uniformity for $G$. This consists of all neighborhoods of the diagonal $\Delta$ [5, p. 197]. Let $A_{\varepsilon}=$ $\{(x, y) \in S \times S:|f(x)-f(y)| \geqq \varepsilon\}$. Then $f$ is uniformly continuous on $S$ iff for every $\varepsilon>0, \operatorname{cl}\left(A_{\varepsilon}\right) \cap \Delta=\varnothing$. Suppose there is an $f \in \operatorname{LUC}(S)$ where $f \notin \mathrm{UC}(S)$. Then there is an $\varepsilon>0$ such that $\operatorname{cl}\left(A_{\varepsilon}\right) \cap \Delta \neq \varnothing$. Hence there is a net $(x(\alpha), y(\alpha))$ in $A_{\varepsilon}$ which converges to $(t, t) \in \Delta$. Now let $\{t(\beta)\}$ be a net in $S$ which converges to $t^{-1}$. Then $\lim _{x, \beta}(x(\alpha) t(\beta))=e=\lim _{x, \beta}(y(\alpha) t(\beta))$. Then by part (ii) above given $\varepsilon>0$ there are $\alpha$ and $\beta$ such that

$$
|f(x(\alpha) t(\beta) s)-f(s)|<\varepsilon / 2 \text { and }|f(y(\alpha) t(\beta) s)-f(s)|<\varepsilon / 2
$$


for all $s \in S$. But $x(\alpha) \in S$ and $y(\alpha) \in S$ hence

$$
\begin{aligned}
|f(x(\alpha))-f(y(\alpha))| \leqq & |f(x(\alpha))-f(y(\alpha) t(\beta) x(\alpha))| \\
& +|f(x(\alpha) t(\beta) y(\alpha))-f(y(\alpha))|<\varepsilon .
\end{aligned}
$$

This contradicts that $(x(\alpha), \nu(\alpha)) \in A_{\varepsilon}$.

COROLLARY 5. If $G$ is a topological group, $S$ is an abelian subsemigroup of $G$ where $\operatorname{cl}(S)$ is compact, then the pair $(S, G)$ has properiy $P$.

Proof. $\operatorname{cl}(S)$ is a compact topological group [3, Theorem 9.16]. So we can make the extension from $S$ to $\operatorname{cl}(S)$. Then by Theorem 3 we can make the extension from $\operatorname{cl}(S)$ to $G$.

Finally we will show that whenever we have an extension, we have a norm preserving extension.

Proposition 6. If $S$ is a subsemigroup of a topological semigroup $T$ and the pair $(S, T)$ has property $P$, then given $f \in \operatorname{LUC}(S)$ there is a $g \in \mathrm{LUC}(T)$ such that $\left.g\right|_{S}=f$ and $\|g\|=\|f\|$.

Proof. Let $F \in \operatorname{LUC}(T)$ be an extension of $f \in \operatorname{LUC}(S)$. If $g=(F \wedge\|f\|) \vee$ $(-\|f\|)$ where $\|f\|$ and $-\|f\|$ are constant functions, then $g \in \operatorname{LUC}(T)$ [8, Lemma 1.1] and is the desired norm preserving extension of $f$.

\section{BIBLIOGRAPHY}

1. N. Bourbaki, Eléments de mathématique. Part 1. Les structures fondamentales de l'analyse. Livre III: Topologie générale, Actualités Sci. Indust., no. 1029, Hermann, Paris, 1947; English trans!., Hermann, Paris; Addison-Wesley, Reading, Mass., 1966. MR 9, $261 ; 34$ \#5044b.

2. E. Granirer and A. Lau, Invariant means on locally compact groups, Illinois J. Math. 15 (1971), 249-257.

3. E. Hewitt and K. A. Ross, Abstract harmonic analysis. Vol. I: Structure of topological groups. Integration theory, group representations, Die Grundlehren der math. Wissenschaften, Band 115, Academic Press, New York; Springer-Verlag, Berlin, 1963. MR 28 \#158.

4. M. Katetov, On real-valued functions in topological spaces, Fund. Math. 38 (1951), 85-91; correction, ibid. 40 (1953), 203-205. MR 14, 304; MR 15, 640.

5. J. L. Kelley, General topology, Van Nostrand, Princeton, N.J., 1955. MR 16, 1136.

6. A. T. Lau, Topological semigroups nith invariant means in the convex hull of multiplicative means, Thesis, Univ. of British Columbia; Trans. Amer. Math. Soc. 148 (1970), 69-84. MR 41 \#1911.

7. T. Mitchell, Topological semigroups and fixed points, Illinois J. Math. 14 (1970), 630-641.

8. I. Namioka, On sertain actions of semi-groups on L-spaces, Studia Math. 29 (1967), 63-77. MR 36 \#6910.

Department of Mathematics, la Salle College, Philadelphia, Pennsylvania 19141 